\title{
Criptococcosis en el Hospital Carlos Van Buren de Valparaíso: una serie de casos
}

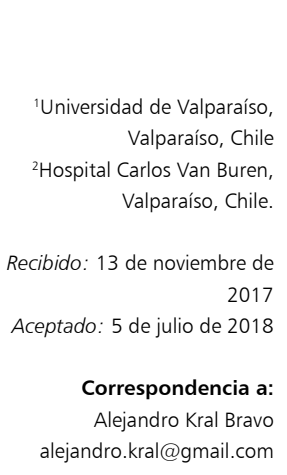

.


En nuestro país no existen publicaciones que permitan conocer las características de los pacientes que desarrollan infección por este agente, ni tampoco sobre su pronóstico a mediano y largo plazo, por lo que surge el interés en identificar y caracterizar a los pacientes que desarrollan infección por este agente, en nuestra realidad local.

El objetivo del siguiente estudio fue caracterizar a los pacientes que presentaron infección por Cryptococcus spp. en el Hospital Carlos Van Buren (HCVB) de Valparaíso.

\section{Pacientes y Métodos}

Mediante el Software Kern-Mic Microbiology se identificaron los cultivos con desarrollo de Cryptococcus spp. en el laboratorio de microbiología del HCVB, entre el 1 de enero de 2013 y el 30 de junio de 2016, que corresponden a la fecha en las cuales se inicia el uso de este programa de registro en el laboratorio, y la fecha en la cual se termina la recolección de los datos. A partir de esta información se obtuvo las fichas clínicas de los pacientes, las que fueron analizadas por dos revisores independientes, registrando la información en una base de datos generada en software Microsoft Excel. Se generaron medidas de resumen utilizando el Software Stata 14.0.

El diagnóstico definitivo de criptocococis se realizó en base a la positividad de los cultivos para cualquier especie del género Cryptococcus, realizado a partir de muestras de líquido cefalorraquídeo (LCR), sangre, orina, lavado bronquio-alveolar (LBA) o líquido pleural. No se utilizó la tinción de tinta china como método diagnóstico para la inclusión de pacientes en el estudio.

Se clasificaron los casos como meningitis criptococócica si presentaban cultivos de LCR positivos; criptococosis diseminada si presentaban hemocultivos con desarrollo de Cryptococcus spp.; y criptococosis pulmonar si la presencia del hongo se encontraba en una muestra de LBA. Todos los pacientes se manejaron hospitalizados.

Se registraron la edad, sexo, y co-morbilidades de los pacientes, además de las características citológicas, bioquímicas y tinción con tinta china del líquido cefalorraquídeo en caso de infección meníngea (junto al recuento de leucocitos, proteínas, glucosa y lactato). La mortalidad o sobrevida de los pacientes se determinó en base a los registros clínicos de los controles ambulatorios al año del episodio infeccioso, y en base a la información obtenida de los certificados de defunción en el registro civil.

\section{Resultados}

Durante el período de estudio, 16 pacientes presentaron cultivos con desarrollo de Cryptococcus spp. Se logró obtener información de sus respectivas fichas clínicas en 13 de ellos, de los cuales dos pacientes habían presentado dos infecciones por este microorganismo, obteniendo un total de 15 casos de infección por Cryptococcus spp.

Respecto a las características basales de los pacientes, (Tabla 1), de los 13 pacientes incluidos, $2(15,4 \%)$ eran de sexo femenino y $11(84,6 \%)$ de sexo masculino. La mediana de edad al momento de la infección fue de 35 años (RIQ: 33-43).

Del total de pacientes, $11(84,6 \%)$ tenían serología positiva para VIH y uno $(7,7 \%)$ presentaba una leucemia linfática crónica (LLC) y otro $(7,7 \%)$ tenía etilismo crónico como patología de base. De los 11 pacientes con infección por VIH, en seis pacientes se hizo el diagnóstico de síndrome retroviral en conjunto con la infección criptococócica, y cinco pacientes tenían un diagnóstico previo de infección por VIH, con abandono de su terapia anti-retroviral. La mediana de LT CD4 fue de 10 céls/ $\mathrm{mm}^{3}$ al momento de la infección (RIQ 9 - 41); con una mediana de carga viral de 8.955 copias ARN/mm 3 (RIQ 4100-116.642).

De los 15 casos, nueve $(60 \%)$ presentaron infección meníngea, cinco $(33,3 \%)$ presentaron criptococosis diseminada, y uno $(6,6 \%)$ presentó infección pulmonar. En todos los casos la cepa correspondió a C. neoformans.

Se registraron nueve casos de meningitis con cultivo de LCR positivo, de los cuales siete $(77,7 \%)$ presentaron examen de tinta china positivo. En el análisis citoquímico del LCR, (Tabla 2), la mediana de leucocitos fue de 15 céls $/ \mathrm{mm}^{3}$ (RIQ 5-55); la mediana de proteinorraquia fue de $60 \mathrm{mg} / \mathrm{dl}$ (RIQ 40-105); la mediana de glucorraquia de $18 \mathrm{mg} / \mathrm{dl}$ (RIQ 8-34); y una mediana de lactato de 33 mg/dl (RIQ: 25-38).

En 12 pacientes $(80 \%)$ se utilizó anfotericina B deoxicolato asociado a fluconazol; un paciente $(6,6 \%)$ recibió monoterapia con fluconazol; en uno $(6,6 \%)$ se desconoce el tratamiento efectuado por haber sido trasladado a otro centro asistencial; y un último caso falleció antes de iniciar tratamiento. La terapia bi-asociada endovenosa con anfotericina $\mathrm{B}$ deoxicolato más fluconazol se utilizó por un rango de 12 a 18 días, con posterior cambio a terapia oral con fluconazol por un período de 8 a 24 semanas.

En sólo nueve pacientes se pudo documentar cultivos de control del LCR, de los cuales seis $(66,6 \%)$ presentaban negativización.

La mediana de días de hospitalización fue de 33 días (RIQ 28-50). En 14 (93,3\%) casos, el lugar de hospitalización fue el servicio de medicina de mediana complejidad, y sólo uno $(7,1 \%)$ requirió hospitalización en unidad de cuidados intermedios.

De los 13 pacientes evaluados, ocho $(53,3 \%)$ se encontraban fallecidos al año de haber presentado la infección, seis de ellos tenían infección por VIH, uno padecía de LLC, y el último tenía antecedente de consumo perjudicial 
de alcohol. El paciente con antecedente de LLC presentó una criptococosis diseminada, mientras que el paciente con antecedente de etilismo crónico se presentó con compromiso meníngeo.

\section{Discusión}

Este grupo de pacientes, predominantemente de sexo masculino, eran de edad media, inmunocomprometidos, principalmente co-infectados con $\mathrm{VIH}$, con bajo recuento de LT CD4 y alta carga viral, presentándose clínicamente con afectación meníngea.

Más de la mitad de los pacientes con infección por virus VIH, debutó con una infección por Cryptococcus neoformans, lo que pone en evidencia que aún deben realizarse mayores esfuerzos para lograr un diagnóstico en etapas más tempranas de la enfermedad.

A pesar del creciente reporte de casos de infección en pacientes inmunocompetentes en países del hemisferio norte, en nuestra serie de casos la infección sólo afectó a pacientes con algún grado de inmunocompromiso. La especie neoformans fue la única identificada; no se identificaron especies distintas a la neoformans, las que han sido descritas en pacientes sin deterioro de su sistema inmune, y con prevalencia en países tropicales y subtropicales.

En concordancia con lo descrito en la literatura científica, la inmunosupresión celular dada por la infección por el VIH, sería el principal factor de riesgo para esta infección micótica. Sin embargo, en esta serie también encontramos dos pacientes con otras causas de inmunosupresión, como son el etilismo crónico y las patologías hemato-oncológicas. Esto concuerda con otras series de casos, como las publicadas por Fica y cols., quienes describen infección por Cryptococcus spp. en pacientes con cirrosis hepática y en pacientes con trasplante renal y uso crónico de corticoesteroides ${ }^{13}$. En Sudamérica, existe una serie publicada por Da Silva y cols., de 40 casos de criptococosis durante dos años de seguimiento en un centro de Medicina Tropical. El 70\% correspondía a pacientes de sexo masculino, la mediana de edad fue de 28 años, 73\% tenían infección por VIH y en $92 \%$ los cultivos de LCR fueron positivos, lo que se concuerda con los hallazgos de nuestra serie local en $\mathrm{HCVB}^{14}$.

Clásicamente, en los pacientes con infección por VIH se describe que la meningitis criptococócica se produce en aquellos que presentan recuentos de LT CD4 < 100 céls $/ \mathrm{mm}^{3}$, siendo en nuestra serie la mediana de LT CD4 de los pacientes coinfectados con VIH, muy por debajo de este valor. En este mismo sentido, existen reportes latinoamericanos con mayor frecuencia en pacientes con VIH con recuentos de LT CD4 $<100$ céls $/ \mathrm{mm}^{3}$, menores de 40 años, con una mortalidad variable según las series, entre 31 y $75 \%$, siendo el consumo de alcohol y valores de $\mathrm{LDH}>400 \mathrm{U} / \mathrm{L}$, factores de riesgo de mortalidad ${ }^{15}$.

En el análisis de las características citoquímicas del LCR, las alteraciones observadas son más bien inespecíficas, sin un claro patrón que pudiese orientar al agente etiológico de la infección, a pesar que en la literatura médica clásicamente se describe una celularidad aumentada con predominio de monocitos, proteínas normales o elevadas y glucorraquia baja o normal ${ }^{7}$, lo que se correlaciona con los hallazgos observados en nuestro estudio.

La sobrevida a los 12 meses observada en nuestro grupo de pacientes, es ligeramente mayor a la descrita en la gran cohorte italiana de más de 4.000 pacientes con infección por VIH y coinfección por Cryptococcus, serie en la que se reporta una sobrevida de $32 \%$ a 12 meses plazo 5 .

El estudio se encuentra limitado en cuanto a sólo ser representativo de una realidad local con un pequeño número de casos, por lo que no es necesariamente un reflejo de la realidad nacional. En este sentido, mientras no existan mayores reportes de casos de criptococosis, será difícil el establecer características generales de los pacientes en los cuales debamos sospechar esta infección.

\section{Conclusiones}

En nuestro medio, la infección por C. neoformans sigue siendo una infección que debe ser sospechada en pacientes con antecedentes de inmunocompromiso, principalmente co-infección por VIH con bajo recuento de LT CD4, y debe ser siempre activamente investigada en este tipo de pacientes solicitando los métodos diagnósticos correspondientes.

\section{Resumen}

Introducción: La criptococcosis es una infección micótica oportunista grave, Cryptococcus neoformans es la principal especie de importancia médica, pudiendo manifestarse como meningitis, neumonía o criptococcemia. Objetivo: Caracterizar a los pacientes con infección por Cryptococcus sp. entre el 01/01/13 y 30/06/16, en el HCVB. Materiales y Métodos: Se identificaron los cultivos con desarrollo de Cryptococcus sp., y a partir de éstos se obtuvo los registros de los pacientes, los que fueron analizados por dos revisores independientes. Resultados: Se recopiló la información de 13 pacientes, que presentaron 15 casos de infección por C. neoformans. De los 13 pacientes, $11(84,6 \%)$ eran de sexo masculino, con una mediana de edad de 35 años. 11 pacientes $(84,6 \%)$ padecían infección por VIH, uno $(7,7 \%)$ tenía el antecedente de leucemia linfática crónica, y uno $(7,7 \%)$ 
de etilismo crónico. De los 15 casos, nueve (60\%) presentaron infección meníngea; cinco $(33,3 \%)$ presentaron criptococcemia sin compromiso del LCR; y uno $(6,6 \%)$ presentó infección pulmonar. De los 13 pacientes, ocho $(53,3 \%)$ se encontraban fallecidos al año de seguimiento.
Conclusiones: La infección por Cryptococcus sp. es una patología que debe ser sospechada en pacientes con inmunodeficiencia de predominio celular. La infección meníngea fue la forma más frecuente de presentación. Persiste presentando una elevada mortalidad.

\section{Referencias bibliográficas}

1.- Moreira T A, Ferreira M S, Ribas R M, Borges A S. Criptococose: estudo clínicoepidemiológico, laboratorial e das variedades do fungo em 96 pacientes. Rev Soc Bras Med Trop. 2006; 39: 255-8. http://dx.doi. org/10.1590/S0037-86822006000300005.

2.- Chrissy M, Floyd L. Is development of a vaccine against Cryptococcus neoformans feasible? PLoS Pathog 2015; 11: 6. https://doi. org/10.1371/journal.ppat.1004843.

3.- Ye F, Xie J X, Zeng Q S, Chen G Q, Zhong S Q, Zhong N S. Retrospective analysis of 76 immunocompetent patients with primary pulmonary cryptococcosis. Lung 2012; 190 : 339-46. doi: 10.1007/s00408-011-9362-8.

4.- Pappas P, Perfect J, Cloud G, Larsen R, Pankey G, Lancaster D, et al. Cryptococcosis in Human Immunodeficiency Virus-negative patients in the era of effective azole therapy. Clin Infect Dis 2001: 33 (5):690-9. DOI: 10.1086/322597.

5.- Chuang Y M, Ho Y C, Chang H T, Yu C J, Yang P C, Hsueh P R. Disseminated cryptococcosis in HIV-uninfected patients. Eur J Clin Microbiol Infect Dis 2008; 27: 307-10. DOI: 10.1007/s10096-007-0430-1.

6.- Antinoti S, Galimberti L, Magni C, Casella A, Vago L, Mainini F, et al. Cryptococcus neoformans infection in a cohort of Italian AIDS patients: natural history, early prognostic parameters, and autopsy findings. Eur J Clin Microbiol Infect Dis 2001; 20: 711-7.

7.- Powderly W. Cryptococcal meningitis in HIVinfected patients. Curr Infect Dis Rep 2000; 2: 352-7.

8.- Chen Ch, Sy H, Lin L, Yen H, Wang S, Chen $\mathrm{W}$, et al. Epidemiological characterization and prognostic factors in patients with confirmed cerebral cryptococcosis in central Taiwan. J Venom Anim Toxins Includ Trop Dis 2015; 21: 12. doi: 10.1186/s40409-015-0012-0.

9.- Bava A, Robles A, Negroni R, Arechavala A, Bianchi M. Estudio de algunos aspectos epidemiológicos de 253 casos de criptococosis. Rev Iberoam Micol 1997; 14: 111-4.

10.- Lin X. Cryptococcus neoformans: Morphogenesis, infection and evolution. Infect Genet Evol. 2009; 9: 401-16. doi: 10.1016/j. meegid.2009.01.013.

11.- Silva V, Díaz M C, Febré N. Chilean Invasive Fungal Infections Group. Invasive fungal infections in Chile: a multicenter study of fungal prevalence and susceptibility during a 1-year period. Med Mycol 2004; 42 (4): 333-9.

12.- Cruz Ch, Piontelli L. Enfermedad fúngica invasora en pacientes de cinco hospitales de la Región de Valparaíso, Chile. 2004 a 2009. Rev Chil Infect 2011; 28 (2): 123-9. http://dx.doi. org/10.4067/S0716-10182011000200004.

13.- Fica A, Soto A, Dabanch J, Pinilla J, Porte L. Criptococosis en pacientes inmunosuprimidos sin infección por VIH/SIDA: un nuevo problema clínico en Chile. Rev Chilena Infectol 2015; 32 (1): 73-9. doi: 10.4067/S071610182015000200015 .

14.- Da Silva B, Freire A, Bentes A, Sampaio I, Santos L, Dos Santos M, et al. Characterization of clinical isolates of the Cryptococcus neoformans - Cryptococcus gatti species complex from de Amazonas State in Brazil. Rev Iberoam Micol 2012; 29 (1): 40-3. doi: 10.1016/j.riam.2011.05.003

15.- Sifuentes Osornio J, Corzo-León D, Ponce de León A. Epidemiology of invasive fungal infections in Latin America. Curr Fungal Infect Rep 2012; 6: 23-34. DOI: 10.1007/s12281-0110081-7. 